\title{
The EUSO@Turlab Project: Results from Phase II
}

\author{
G. Suino*, H. Miyamoto, M. Bertaina, R. Casu, G. Cotto, R. Forza, M. Manfrin, \\ M. Mignone, R. Mulas, M. Onorato, A. Youssef \\ Department of Physics, University of Torino \& INFN Torino - Italy \\ E-mail: gregoriosuinolgmail.com \\ R. Caruso, G. Contino, N. Guardone \\ Department of Physics and Astronomy, University of Catania \& INFN Catania - Italy
}

\author{
S. Bacholle, P. Gorodetzky, A. Jung, E. Parizot, G. Prevôt \\ APC, Univ Paris Diderot, CNRS/IN2P3 - France
}

P. Barrillon, S. Dagoret-Campagne, J. Rabanal Reina

LAL, Univ Paris-Sud, CNRS/IN2P3, Orsay - France

S. Blin

Omega, Ecole Polytechnique, CNRS/IN2P3, Palaiseau - France

\section{for the JEM-EUSO Collaboration}

The TurLab facility is a laboratory, equipped with a $5 \mathrm{~m}$ diameter and $1 \mathrm{~m}$ deep rotating tank, located in the Physics Department of the University of Turin. Originally built mainly to study problems where system rotation plays a key role in the fluid behaviour such as in atmospheric and oceanic flows at different scales, in the past few years the TurLab facility has been used to perform experiments related to observation of Extreme Energy Cosmic Rays from space using the fluorescence technique, as in the case of the JEM-EUSO mission, where the diffuse night brightness and artificial light sources can vary significantly in time and space inside the Field of View of the telescope. The description of the EUSO@TurLab project and its first results have been presented in the past. During the last two years many upgrades have been performed on the instrumentation mainly related to the read-out electronics: SPACIROC-1 (employed in EUSO-Balloon and EUSO-TA prototypes) and SPACIROC-3 (EUSO-SPB and Mini-EUSO) which allowed to test a fully equipped Elementary Cell of JEM-EUSO. This phase has been named Phase II. Moreover, the Focal Surface of EUSO-Balloon with the level 1 trigger logic implemented in the PhotoDetector Module has been tested at TurLab after the Canada flight. Finally, tests related to the possibility to employ a EUSO-like detector for other type of applications such as wave monitoring and imaging detector have been pursued. The tests and results obtained in EUSO@TurLab Project - Phase II are described.

35th International Cosmic Ray Conference - ICRC2017 - 10-20 July, 2017 Bexco, Busan, Korea

${ }^{*}$ Speaker. 


\section{Introduction and TurLab facility}

The TurLab [1] laboratory (denoted TurLab in the following) is a facility used in preparatory steps of the JEM-EUSO project [2]. The aim of JEM-EUSO is the detection of fluorescence and Cherenkov light produced by Extensive Air Showers (EAS) generated by Extreme Energy Cosmic Rays (EECRs, energy E $>5 \times 10^{19} \mathrm{eV}$ ) impinging the Earth's atmosphere. Recognising the highly-variable luminous conditions of the atmosphere is the main task of the trigger system, and simulations of different background conditions have been made to implement and improve the trigger logic.

JEM-EUSO Focal Surface (FS) consists of Hamamatsu 64-ch Multi-Anode PhotoMultiplier Tubes (MAPMTs). One Elementary Cell unit (EC unit) consists of 4 PMTs, and 9 EC units form a Photo-Detector Module (PDM). In total, the entire focal surface consists of 137 PDMs with about 5,000 PMTs with 0.3M pixels. The output of PMTs are readout by an Elementary Cell ASIC board (EC ASIC) which consists of 6 SPACIROC ASICs [3]. A PDM board is an interface board between a PDM and the latter part of data processing system, which sends slow control commands and processes First Level Triggers (FLTs).

The use of TurLab gives the opportunity of reproducing experimentally variable luminosity conditions and different possible light sources, recreating orbit sights and events on the JEM-EUSO scale. For this reason, TurLab represents an ideal condition for testing JEM-EUSO FS elements, electronics and trigger system at real working conditions. In the following we summarize the experimental setup used for the above purposes. Details can be found in [1]. TurLab is equipped with a $5 \mathrm{~m}$ diameter rotating tank with rotation period ranging from 12 seconds to 20 minutes, located $15 \mathrm{~m}$ below ground level; therefore, without artificial illumination, the room is much darker than the night sky. In the EUSO@TurLab project we use the TurLab rotating tank with a series of different configurations to recreate the Earth views and an imaging detector hung from the ceiling above the tank pointing towards nadir to mimic the JEM-EUSO telescope (see Fig. 1). The original idea of using this facility in the framework of JEM-EUSO was to collect and process data to verify the response of the FLT. Details of the FLT logic can be found in [4]. The phenomena observed in the tank, such as waves, gave hints also of very different applications that would be interesting to study further.

Fig. 2 shows the conceptual figure of EUSO FLT (top) with an example of an event taken using the EUSO-Balloon instrument with the aim of mimicking cosmic ray-like events [5]. The pixels in the MAPMT are subdivided in overlapping boxes of $3 \times 3$ pixels. A threshold is set at pixel level, based on the average number of background counts, coming mainly from UV nightglow. The sum of the counts in excess in each box for each time frame, called Gate Time Unit (GTU), are compared with a pre-fixed value $(S)$ also variable according to the background level. Only signals in excess provide a valid trigger. In the specific case the value of $S$ is placed around the level shown by the dotted blue line on the bottom plot.

By means of this rotating tank in the dark environment, we have been testing EUSO electronics such as its basic performance as well as FLT for cosmic rays in the various background and sceneries which are transitting from one to another.

While in the first phase of the TurLab experiments we used only one MAPMT and a read-out electronics based on standard electronics (NIM and CAMAC), during TurLab phase II, the detector 


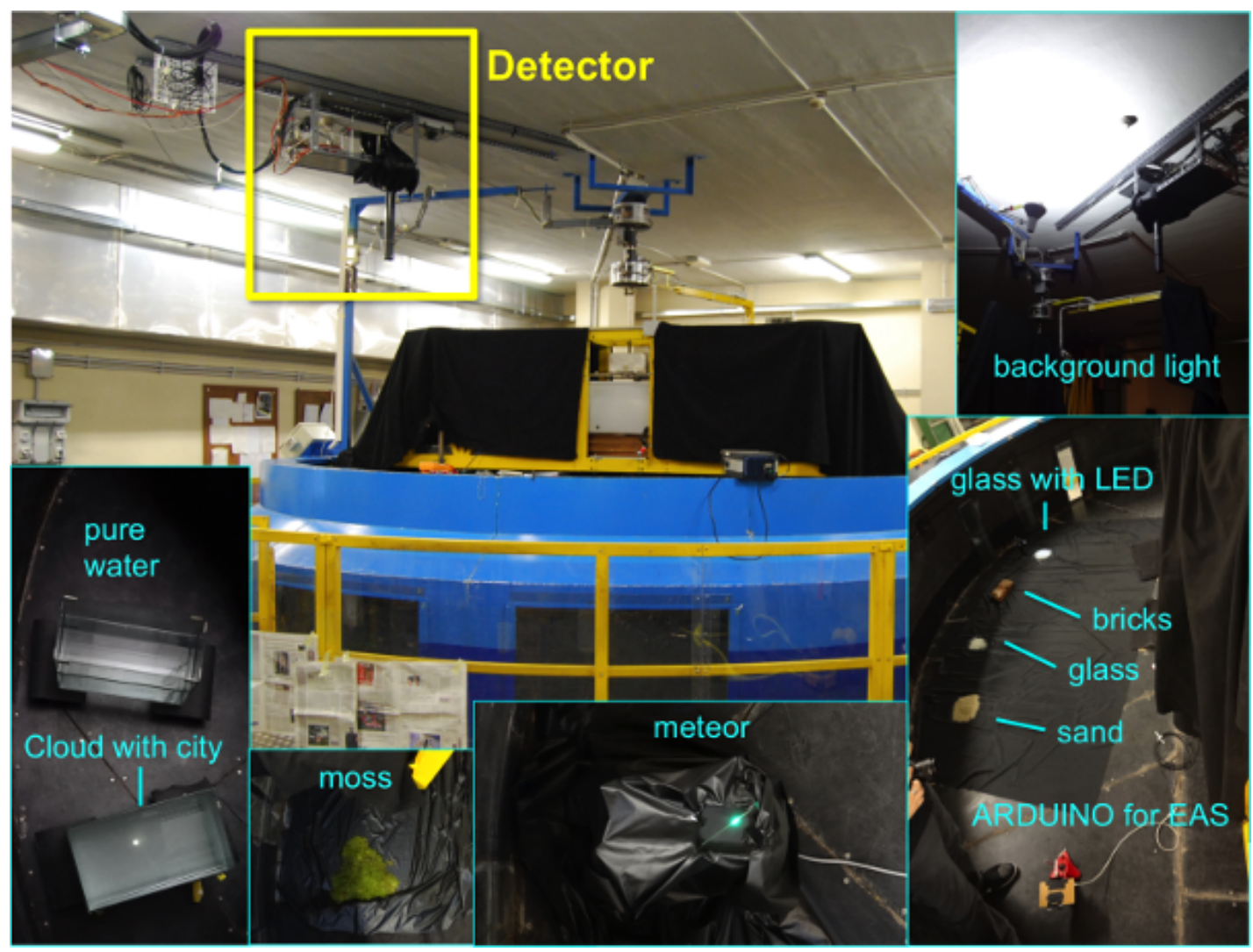

Figure 1: The TurLab rotating tank. The black tube on the ceiling, within the yellow box indicating the detector, shows the collimator of the experimental setup used to mimic the JEM-EUSO telescope. Light sources and materials used to mimic other phenomena are shown as well.

improved being equipped by one full EC and relative electronics. In this way, we could test the trigger logic directly on the hardware foreseen for JEM-EUSO and its path-finder missions. It consists of a $2 \times 2$ array of 64 -ch MAPMTs read by SPACIROC ASICs. When the EUSO EC_unit camera is set on the ceiling at the height of $2 \mathrm{~m}$ above TurLab tank, we know that a pixel has a FoV of $5 \times 5 \mathrm{~mm}^{2}$ from the past measurements. This FoV corresponds to a solid angle of $6.25 \times 10^{-6} \mathrm{sr}$ which is comparable to the one for JEM-EUSO.

Figure 1 shows the TurLab tank, light sources and materials used to mimic the various kinds of phenomena and albedos that JEM-EUSO will encounter. We use an EUSO EC_unit with a lens as a camera, readout by the EUSO front-end electronics with a test board, observing several materials mimicking phenomena such as meteor, cosmic ray, cloud, 'city lights', passing from one to another in the FoV in a constant background light produced by a high power LED lamp diffused on the ceiling. High voltages, DC power supplies, function generators and monitoring oscilloscopes are on the desk by the side of the tank with a PC with LabView interface for the test board to send slow control and data acquisition, and ROOT programs for monitoring and analysis. Fig. 3 shows the examples of UV images obtained by the EC unit camera during the full tank rotation.

The top panel of Fig. 4 shows the result of UV intensities in a full rotation of the tank with various materials. The plot shows the summed counts of a PMT (=64 pixels) as a function of time 


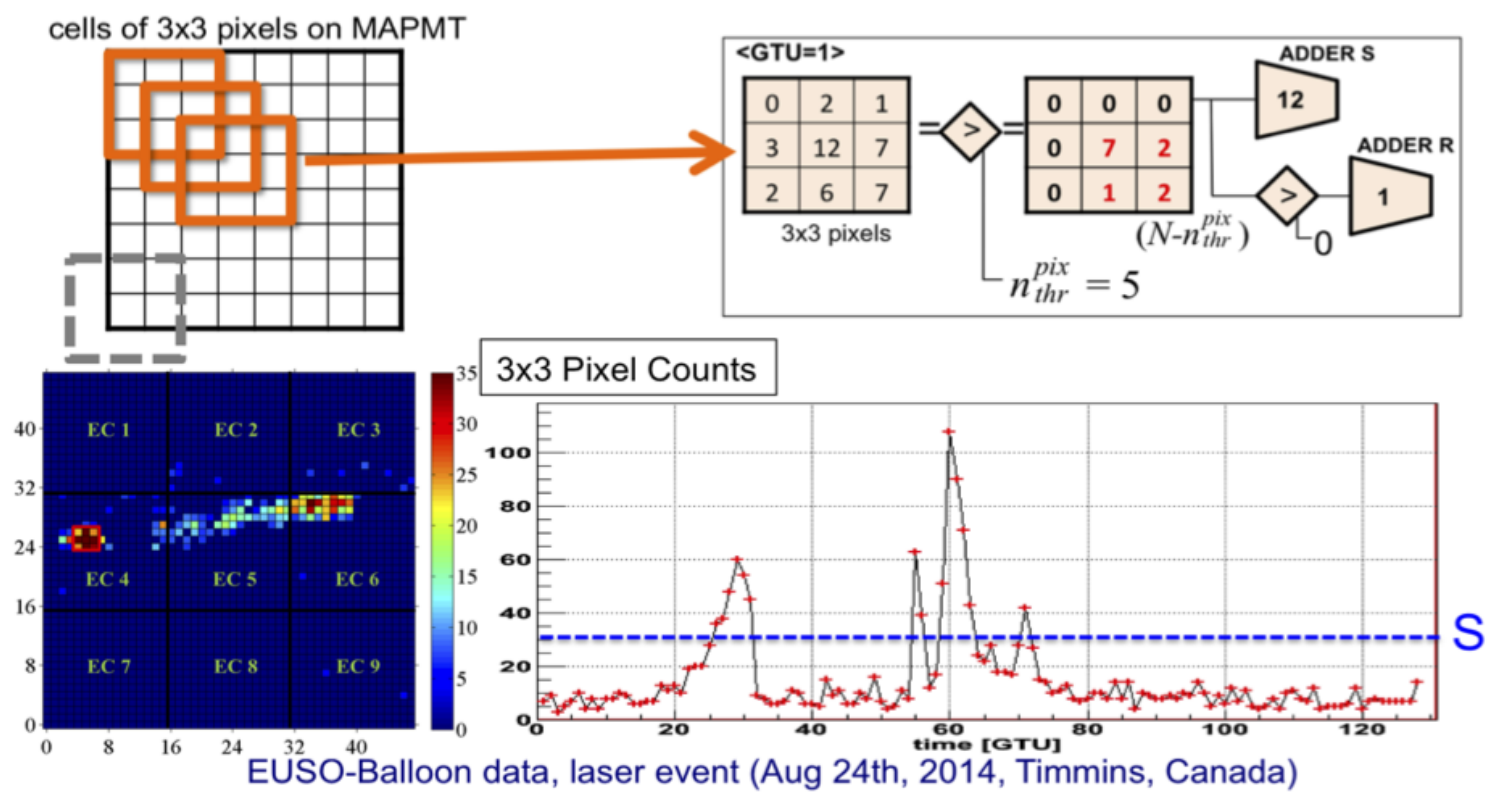

Figure 2: Top: Conceptual figure of FLT. Bottom: integrated image (left) and $3 \times 3$ pixel counts (right) during an EUSO-Balloon laser event mimicking a $\mathrm{CR}$ event. The $S$ value indicates the minimum number of pixel counts in a box within $n_{t h r}^{p i x}$ GTUs to be triggered.

in unit of frames. Similar results were obtained also by the other three MAPMTs. Afterward, we analysed the data and processed the FLT offline. The middle panel shows the averaged counts per pixel which is used to set the FLT thresholds, while the bottom shows when FLTs were issued based on signals in that PMT, as a function of frame (GTU). Almost all triggers coincide with passing over the Arduino driven LED chain, which mimics cosmic-ray like events, as it should be. Only in few cases the triggers occurred not in presence of the cosmic-ray events. A careful inspection of the situation clarified that these events occurred in presence of very fast variable conditions of the reflection of the tank. Such events are detected because the read-out system is capable of memorizing only 100 GTUs $(250 \mu)$ every $\sim 50 \mathrm{~ms}$. In case of JEM-EUSO this would not be the case. This was confirmed experimentally by the tests described in Section 2.

\section{PDM tests at TurLab}

After verifying offline the performance of the trigger logic, the VHDL code was implemented in the FPGA on board the PDM to check the performance of the same logic in hardware. In this case the PDM employed in the EUSO-Balloon campaign [5] was hung on the ceiling with all its data acquisition system, and the FLT was tested. As intended by design, the FLT was successfully issued by the PDM control board electronics in the case of the cosmic-ray-like events emulated by the Arduino-controlled LEDs, but not in the case of the slower events representing a meteor track (simulated with the oscilloscope), nor on the permanent city lights. To reach a higher level of confidence in the experimental tests, the map of the city of Turin and its surrounding was reproduced artificially as shown in fig. 5 (right side). The map was reproduced at the same scale each pixel of 


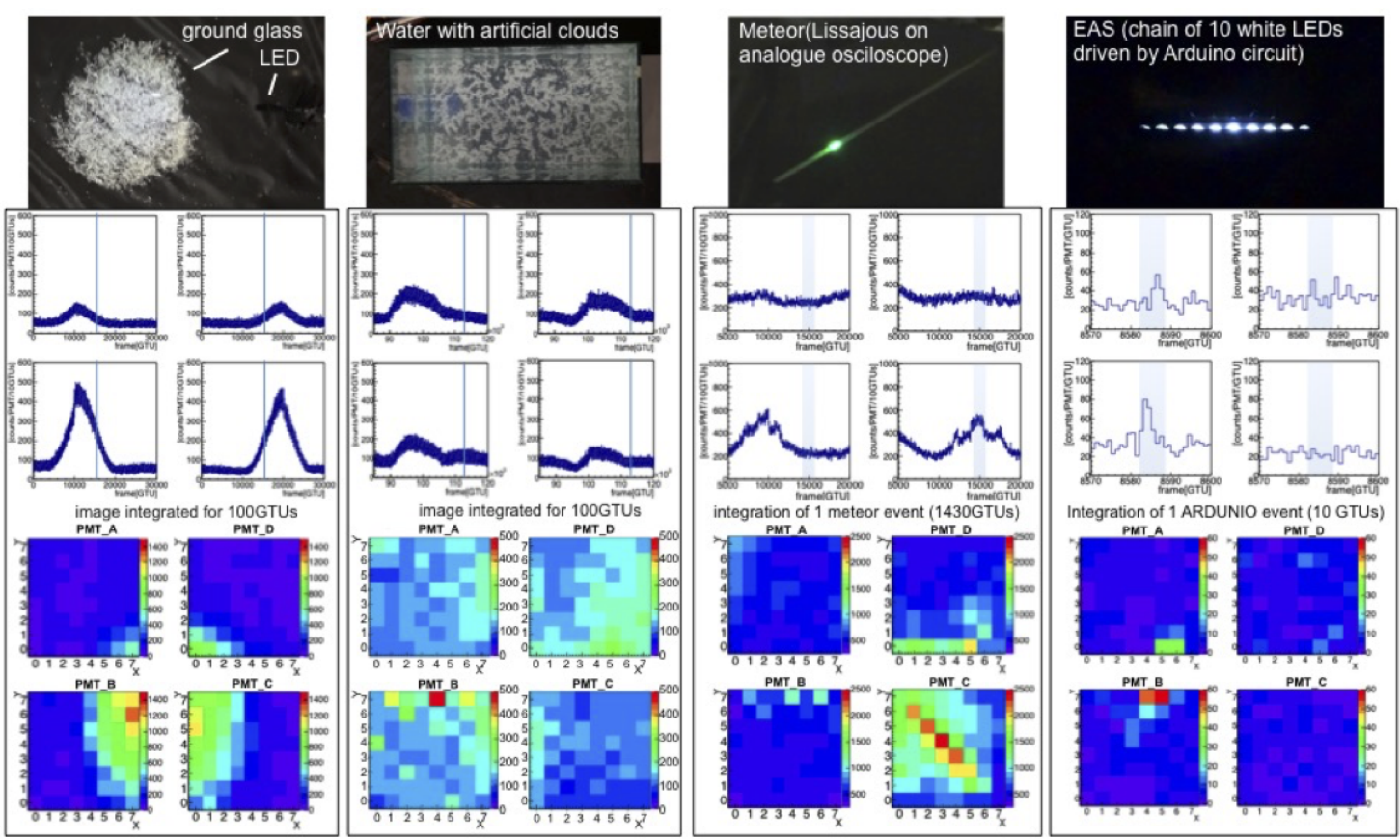

Figure 3: Examples of UV images obtained by the EC unit $(2 \times 2$ MAPMTs $)$ camera passing by the various materials during the full tank rotation $(9 \mathrm{~min})$. Starting from the left, 'city light' obtained by a ground glass illuminated by LED, cloud, meteor and EAS like events are mimicked as shown in the photos on the top. Middle plots show the summed counts per each PMT per appropriate frame as a function of frame (GTU), while bottom plots show the images obtained during each event integrated during the frames indicated by blue vertical lines in middle plots.

JEM-EUSO would see from the International Space Station. The rate of fake triggers was at $\mathrm{Hz}$ level, which is well below the requirements of JEM-EUSO which sets the limit for the FLT trigger rate to $7 \mathrm{~Hz}$. Another important result was the confirmation that the presence of a city or another artificial light source in some part of the telescope's FoV does not prevent the instrument from detecting cosmic-ray showers in a different part of the FoV, as shown in Fig. 5. This was tested with a cosmic-ray-like event just beside the replica of Turin city lights. The FLT trigger was issued nominally, demonstrating the independence of the MAPMTs, even within the same EC. The ability to detect a cosmic-ray event even in the presence of intense light nearby is crucial to maximize the duty cycle and acceptance of the JEM-EUSO instrument.

\section{Other applications}

TurLab is a laboratory for geo-fluid-dynamics studies, where rotation is a key parameter such as Coriolis force and Rossby Number. With using inks or particles, based on the fluid-dynamics, key phenomena such as planetary atmospheric and fluid instabilities can be reproduced in the TurLab water tank. We took, therefore, the opportunity to study the response of our detector in presence of waves and/or foam inside the tank. What we noticed was the change in the UV reflection if water had patches of foam. This result is interesting because it gives hints that a UV-sensitive 


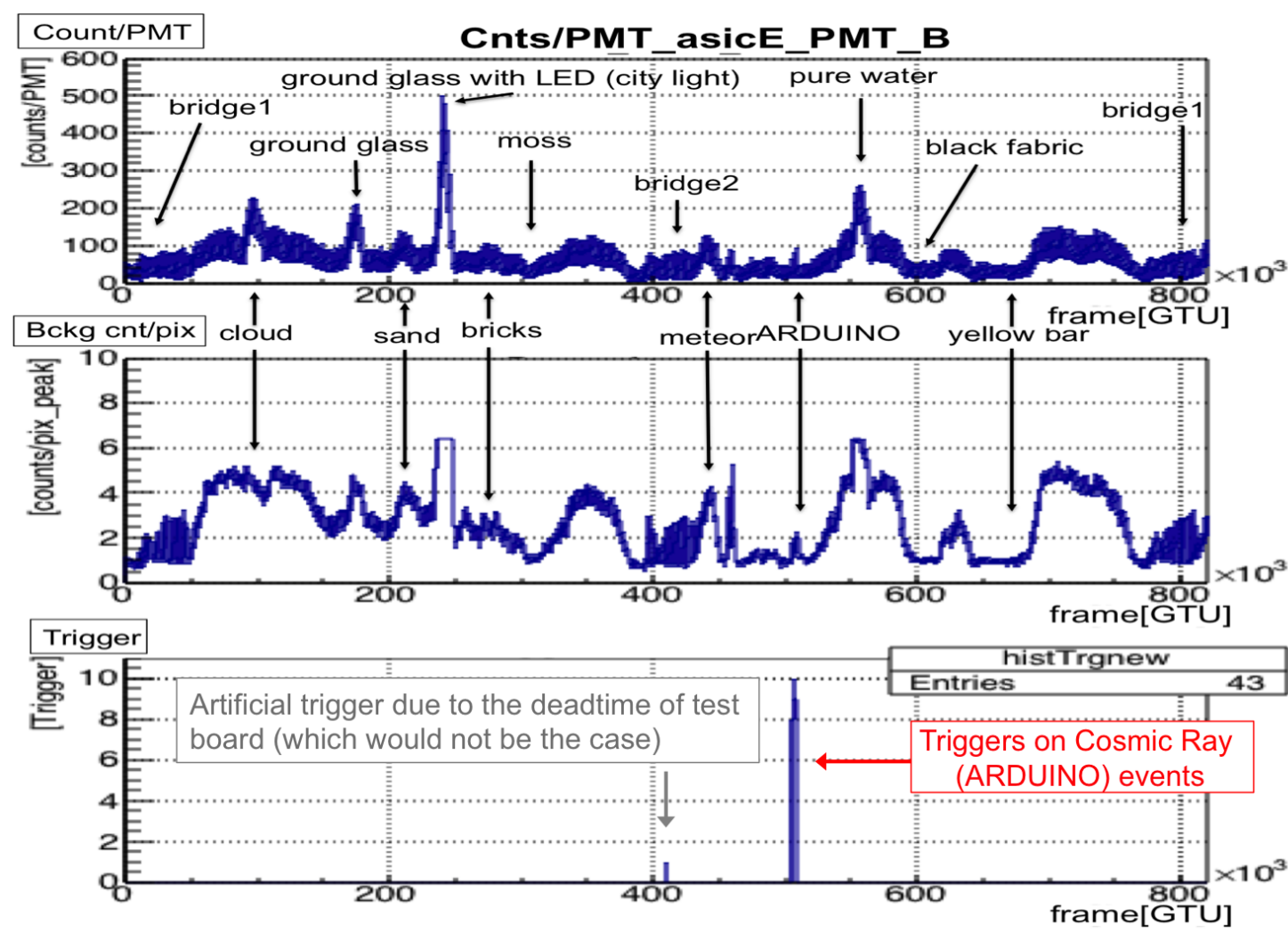

Figure 4: The top panel shows the raw data (counts/PMT), while the middle shows the corresponding background level which is used to set the threshold for the FLT and the bottom shows the result of the FLT, as a function of frame $(\mathrm{GTU}=2.5 \mu \mathrm{s})$ respectively. We obtained $100 \%$ of the trigger efficiency for the cosmic-ray-like events. A few triggers at around frame 410,000 are due to about $50 \mathrm{~ms}$ of dead-time for using the test board, which would not be the case of actual EUSO observation operated with the PDM board with no dead-time.

flying detector could be used to monitor sea condition. Whitecaps are a major source for wave energy dissipation and represent an important mechanism for air-sea exchange of gasses. Bubble bursting produces marine aerosols that contribute to climate regulation and whitecaps are reflector of light radiation responsible for increase of albedo.

Another interesting experimental study performed at TurLab was the study of the effect of the reflection of light from waves. The tank was filled with $1 \mathrm{~m}$ of water and industrial fans were used to generate sea waves. A lamp was illuminating the water and the reflected light on the ceiling was retrieved by the MAPMTs and stored on disk. Inside the tank, water dedicated probes were measuring the height of waves. A Fourier analysis of the wave height and of the light intensities showed that peaks occurred at the same frequencies (see Fig. 6). This indicates that the intensity of the light was correlated with the wave height. The two results here described might indicate that JEM-EUSO could be used to observe ocean phenomena. This will be tested by analizing the data collected by EUSO-SPB [7] during its flight campaign of April-May 2017. 


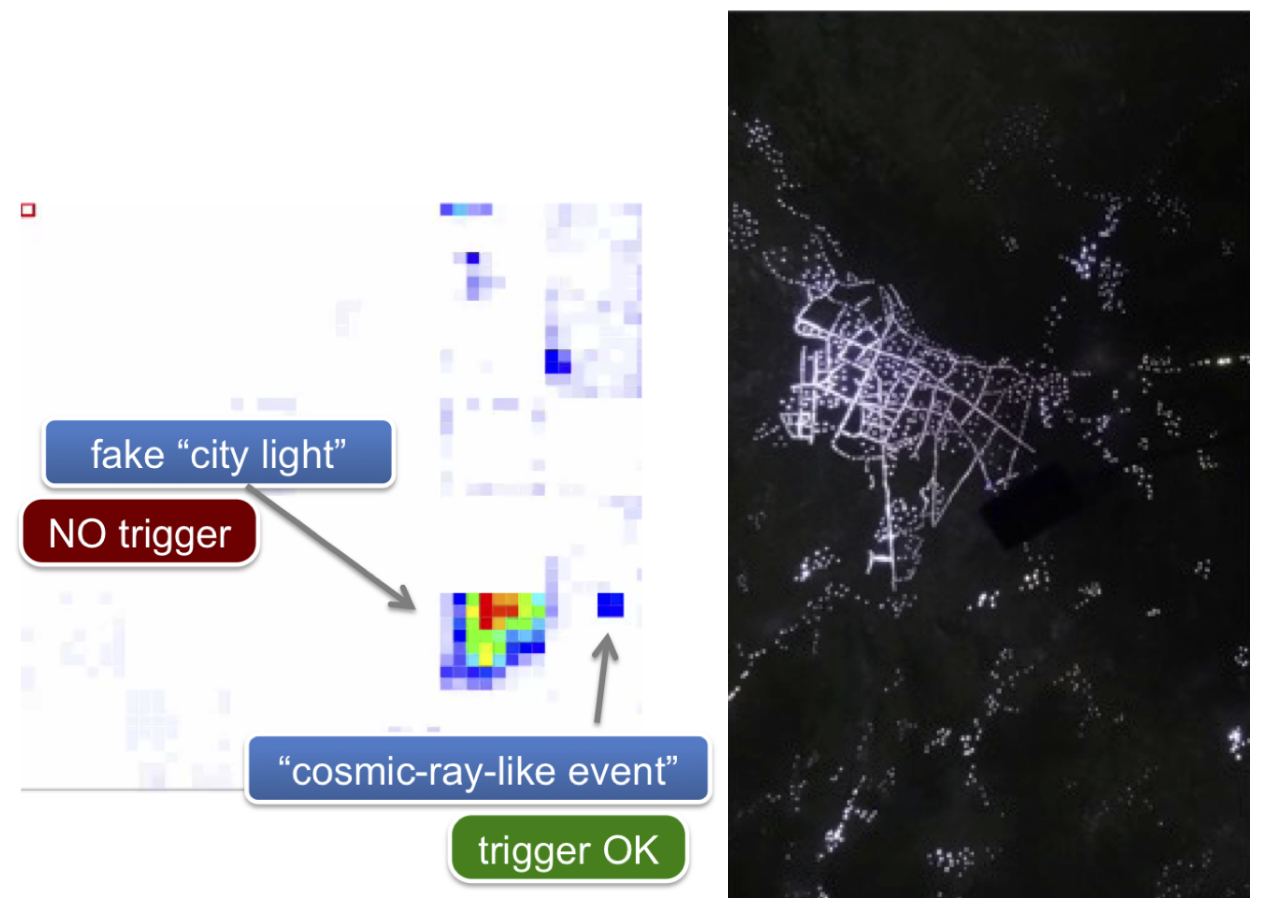

Figure 5: Map of the photon counts in the $48 \times 48$ pixel array of the PDM installed at the Turlab facility. The image shows the strong light of permanent LEDs representing a city which is diplayed on the right side of the figure, and a fainter spot of 4 pixels beside them, corresponding to a light signal representing a cosmic-ray shower. The red color indicates count values exceeding 100, while the blue one covers the range up to 20 counts. Both 'city' and 'cosmic-ray' are viewed by the same EC but on different MAPMTs. For this reason the 'cosmic-ray' is properly triggered.

\section{Conclusions}

The measurements presented in this paper represent the second successful step of an on-going project aiming at using TurLab as a facility to mimic experimentally transient lights that JEMEUSO and its path-finder missions are expected to see during their orbits on the ISS or from the edge of the atmosphere.

The data taken so far have been used to validate the FLT logic. The system, that automatically adjusts the thresholds to keep the rate of triggers on background fluctuations below $1 \mathrm{~Hz} / \mathrm{EC}$ even in case of slow background variations, is effective. The FLT detects EAS-like events with light intensities comparable to those JEM-EUSO would observe in the expected energy range (E $>$ $5 \cdot 10^{19} \mathrm{eV}$ ) and in presence of the expected nightsky background intensities. The FLT has shown to be quite effective in rejecting city-like and lightning type of events. Only few spurious triggers occur. The TurLab has been used also to test the FLT implemented directly on a PDM and proved to work as expected. TurLab gave insights on the possibility to apply the JEM-EUSO observation principle to detect other type of phenomena such as ocean conditions, using dedicated triggering system. Recently, the TurLab facility is being used to test the trigger logic foreseen for MiniEUSO [6]. The results of these tests are subject of a different publication [8]. 

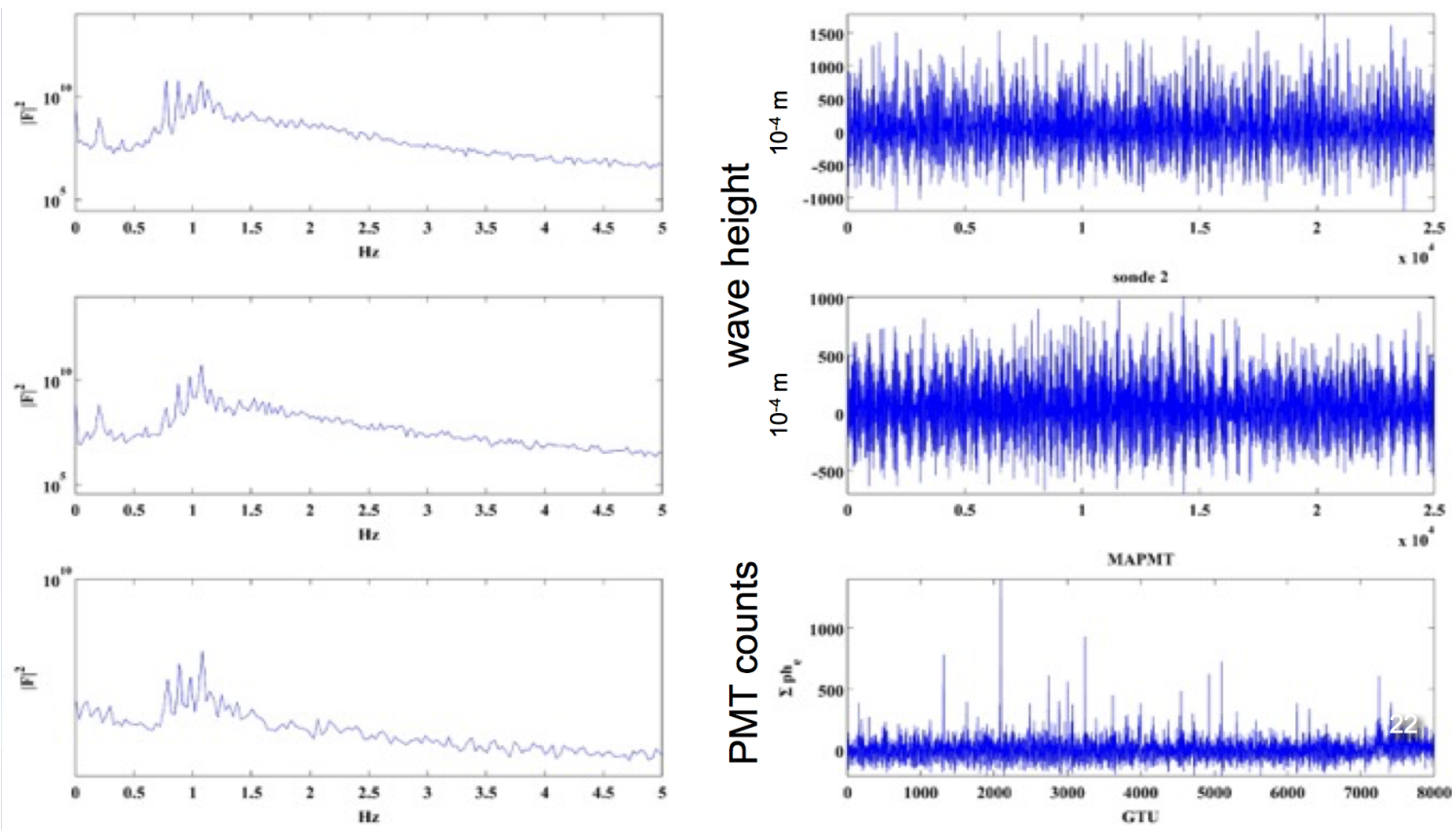

Figure 6: Right side: Top and middle plot show the wave heights measured with dedicated probes as a function of time. Bottom plot show the MAPMT counts recorded at similar times due to the reflected light of a lamp illuminating the water. Left side: Fourier analysis of the plots on the right side. Both probes and MAPMT intensities show peaks at similar frequencies.

\section{Acknowledgments}

This work has been partially funded by the Italian Ministry of Foreign Affairs and International Cooperation and by the European High-Performance Infrastructures in Turbulence (EuHIT).

\section{References}

[1] M. Bertaina et al. (JEM-EUSO Coll.), EPJ Web of Conferences 89 (2015) 03003.

[2] T. Ebisuzaki et al, Adv. Sp. Res. 53 (2014) 1499.

[3] H. Miyamoto et al., PoS TIPP2014 (2015) 362.

[4] G. Abdellaoui et al. (JEM-EUSO Coll.), Nucl. Instr. \& Meth. A 866 (2017) 150.

[5] J. Eser et al. (JEM-EUSO Coll.), Proc. 34th ICRC, Den Haag, \#860 (2015).

[6] M. Ricci et al. (JEM-EUSO Coll.), "Mini-EUSO: a precursor mission to observe and study Atmosphere and Earth UV emission from the International Space Station", This Conference Proceedings, Busan, (2017).

[7] L. Wiencke et al. (JEM-EUSO Coll.), "EUSO-SPB Mission and Science", This Conference Proceedings, Busan, (2017).

[8] F. Capel et al. (JEM-EUSO Coll.), "The Mini-EUSO multilevel trigger algorithm and its performance", This Conference Proceedings, Busan, (2017). 